\title{
STUDY OF THE PERMANENT CONJECTURE AND SOME GENERALIZATIONS
}

\author{
BY O. S. ROTHAUS ${ }^{1}$ \\ Communicated by Harry Kesten, April 6, 1972
}

Let $K$ be a convex polyhedron in an affine space with a set of extreme points $\mathscr{E}$. A set $\mathscr{S}=\left\{A_{1}, A_{2}, \ldots, A_{n}\right\}$ of affine functions, none of them identically zero on $K$, is said to determine $K$ if there exists an affine subspace $H$ such that $K=\left\{x \in H \mid A_{i}(x) \geqq 0 \forall_{i}\right\}$. Let $R_{0}^{n}$ be closed first $2^{n}$-gant in $\boldsymbol{R}^{n} . \boldsymbol{R}_{0}^{n}$ is a multiplicative semigroup and for two points $u$ and $v$ in $R_{0}^{n}$ we define additionally $u^{v}=u_{1}^{v_{1}} \cdot u_{2}^{v_{2}} \ldots u_{n}^{v_{n}}$. $0^{0}=1$. If $\alpha$ is a scalar $\geqq 0$, we define $u^{\alpha}=\left(u_{1}^{\alpha}, u_{2}^{\alpha}, \ldots, u_{n}^{\alpha}\right)$. Also we define a map $A: K \rightarrow R_{0}^{n}$ by $A(x)=\left(A_{1}(x), A_{2}(x), \ldots, A_{n}(x)\right)$. Let $c$ be a strictly positive function on $\mathscr{E}$. For $y \in \boldsymbol{R}_{0}^{n}$, define

$$
Q(y)=\sum_{e \in \mathscr{E}} c(e) y^{A(e)}
$$

and, for $x \in K$, define $P(x)=Q(A(x))$. $P$ is strictly positive, so it is of some interest to find its minimum.

If $K$ is the set $D_{k}$ of $k \times k, k \geqq 2$, doubly stochastic matrices, and we take for $\mathscr{S}$ the coordinate functions and take $c \equiv 1, P(x)$ is the permanent of $x, \operatorname{Perm}(x)=\sum x^{\pi}$, where the summation is over the permutation matrices.

Returning now to the general case, define a map $q: R_{0}^{n} \rightarrow K$ by

$$
q(y)=\frac{1}{Q(y)} \sum_{e \in \delta} c(e) y^{A(e)} e,
$$

defined for $Q(y) \neq 0$, and a map $h: K \rightarrow K$ by $h(x)=q(A(x))$. Then

THEOREM 1. $h$ is a bijection.

$Q$ is homogeneous of degree $d$ if $\sum_{i} A_{i}(e)=d$ for all $e \in \mathscr{E}$. Since the sum of the inner normals to the faces of $K$, with lengths equal to the area of the faces, is zero, homogeneity can always be achieved by appropriate choice of $\mathscr{S}$. In case $Q$ is homogeneous, we have additionally

THEOREM 2. $P(h(x)) \geqq P(x)$, with equality only for $h(x)=x$.

AMS 1970 subject classifications. Primary 15A15, 52A25; Secondary 05A20.

Key words and phrases. Permanent conjecture, convex polyhedra.

${ }^{1}$ This research was partially supported by NSF GP-8129.

Copyright (C) American Mathematical Society 1972 
The permanent is homogeneous of degree $k$, so the map $h$ above is of considerable interest. For one thing, we have the amusing statement that every $y \in D_{k}$ may be written in the form

$$
y=\sum_{\pi} x^{\pi} \pi / \sum_{\pi} x^{\pi}
$$

for a unique choice of doubly stochastic $x$. It would be interesting to obtain an intrinsic characterization of the representation of $y$ above in the class of all representations of $y$ as a convex sum of permutation matrices. For another, the inverse to the map $h$ does not increase the permanent, but except for the case $k=2$, it appears difficult to get useful expressions for the inverse. There is another description of the map $h$ which is also useful. Let $x \in D_{k}$, and let $X_{i j}$ be the $k-1$ by $k-1$ minor associated to $x_{i j}$. Then

$$
h_{i j}(x)=\frac{x_{i j} \operatorname{Perm}\left(X_{i j}\right)}{\operatorname{Perm}(x)} .
$$

In the study of the homogeneous case, one is led quite naturally to the following considerations. Let $L$ be the set of $l \in R_{0}^{n}$ such that $l^{A(e)} \geqq 1$ $\forall e \in \mathscr{E}$, which is the same as saying $l^{A(x)} \geqq 1 \forall x \in K$. $L$ is a multiplicative semigroup, but also a convex set, owing to concavity of the logarithm. Define $L_{1} \subset L$ as the set of $l$ such that $l^{L(e)}=1 \forall e \in \mathscr{E} . L_{1}$ is then a group, and it may be proved that $L_{1}$ is the set of extreme points of $L$, but $L$ is not necessarily the convex hull of $L_{1}$. For $y \in R_{0}^{n}$ define

$$
E(y)=\frac{1}{d} \min _{l \in L} \sum_{i} y_{i} l_{i} .
$$

The minimum is always achieved as a matter of fact for a point of $L_{1}$, or as the limit along a sequence of points of $L_{1}$.

THEOREM 3. (i) If $Q(y)=0, E(y)=0$, otherwise

$$
E(y)=\left[Q(y) / P\left(h^{-1}(q(y))\right)\right]^{1 / d} .
$$

(ii) For $u, v \in R_{0}^{n}, E(u+v) \geqq E(u)+E(v)$ and $E\left(u^{p} v^{q}\right) \leqq E^{p}(u) E^{q}(v)$, $p+q=1, p, q \geqq 0$.

The last two properties follow from the definition and the semigroup property of $L$. From (i) we see that $E(y)$ is continuous and that $E(A(x)) \equiv 1$ for $x \in K$.

It is of interest to investigate the cases when $E(y)$ is an attained minimum. To this end we say $y$ has $K$-like support if there exists $x \in K$ such that $y_{i}=0$ if and only if $A_{i}(x)=0$. We say $x \in K$ is indecomposable if, for 
each $i, \exists e \in \mathscr{E}$ for which $A_{i}(e) \neq 0$ and such that $\prod_{v \neq i} A_{v}(x)^{A_{v}(e)} \neq 0$. $y$ with $K$-like support is called indecomposable if the associated $x \in K$ is indecomposable.

THEOREM 4. If $y$ has $K$-like support, $\sum y_{i} l_{i}$ has an attained minimum, and $l_{i}$ is uniquely determined if $y_{i} \neq 0$. (With minor qualifications, the converse of the last statement is also true.) If $y$ is indecomposable, the minimum is uniquely attained.

By examining the properties of the attained minimum, we have

THEOREM 5. If $y$ has $K$-like support, there exists a positive constant $\alpha$, an $l \in L_{1}$, and unique $x \in K$ such that $y=\alpha A(x) l$, and $\alpha=E(y)$. If $y$ is indecomposable, $l$ is also unique.

From this there follows

THEOREM 6.

$$
E(y)=\max _{x \in \mathbf{K}}\left[y^{A(x)} / A(x)^{A(x)}\right]^{1 / d}
$$

Also,

THEOREM 7. If $M$ and $m$ are the upper and lower bounds for $P(x)$, then $M E^{d}(y) \geqq Q(y) \geqq m E^{d}(y)$.

For $D_{k}$, the elements of $L_{1}$ are just matrices whose $i, j$ entry is $\lambda_{i} u_{j}$ with $\prod_{i} \lambda_{i}=\prod_{i} u_{i}=1$. And $x$ is indecomposable if it may not be written as a reduced matrix after some permutation of the rows and another of the columns. The above reduces to the known theorem that if $y$ has the same support as a doubly stochastic matrix, there exist positive diagonal matrices $D_{1}$ and $D_{2}$ such that $D_{1} \times y \times D_{2}$ is doubly stochastic, where the multiplication in last is matrix multiplication.

If $Q(y)=\sum c(e) y^{A(e)}$ is homogeneous of degree $d \leqq 1$, then it is easy to see that $Q(y)$ is a concave function, since each of the summands is, so the minimum of $P(x)$ is attained at an extreme point. If $d \geqq 1$, define

$$
Q_{1}(y)=\sum c(e) y^{A(e) / d}
$$

so that we have $Q_{1}(A(x)) \geqq \min _{e \in \mathscr{\delta}} c(e) A(e)^{A(e) / d}=\lambda$.

Now, for $x \in K$, write $A(x)^{1 / d}=\alpha^{1} A(y)$ with $y \in K, l \in L_{1}, \alpha>0$, and then $\alpha=E\left(A(x)^{1 / d}\right)=1 / E^{1 / d}\left(A^{d}(y)\right)$. Every $y \in K$ occurs for some $x \in K$. Hence

$$
Q_{1}(A(x))=\sum c(e) \alpha^{d} A(y)^{A(e)}=\alpha^{d} P(y),
$$

so $P(y) \geqq \lambda E\left(A^{d}(y)\right)$. Let $I$ be the point in $\boldsymbol{R}_{0}^{n}$ with all coordinates equal 1 . Then

$$
1=E(A(y))=E(A(y) \cdot I) \leqq E^{1 / d}\left(A^{d}(y)\right) E^{(d-1) / d}(I),
$$


so

$$
E\left(A^{d}(y)\right) \geqq \frac{1}{E^{d-1}(I)}=\min _{x \in K}\left[A(x)^{A(x)}\right]^{(d-1) / d} \geqq\left(\frac{d}{n}\right)^{d-1}
$$

THEOREM 8. $P(x) \geqq \lambda\left(\frac{d}{n}\right)^{d-1}, x \in K$.

THEOREM 9. $\operatorname{Perm}(x) \geqq 1 / k^{k-1} ; x \in D_{k}$.

This is a far cry from the van der Waerden conjecture, but better, I believe, than other available results.

By purely combinatorial arguments we can obtain a result in some respects better than the last. Let $\beta=$ integral part of $k^{(k-1) / k}$. Then the sum of the $\beta$ largest terms in the expansion of $\operatorname{Perm}(x), x \in D_{k}$, is $\geqq \beta / k^{k}$, with equality only for the matrix with all equal entries.

If the permanent conjecture is true, then it follows as well that $\operatorname{Perm}\left(x^{r}\right)$, $r \geqq 1$, achieves its minimum at the matrix with all equal entries. Using the mapping function $h$ associated to $\operatorname{Perm}\left(x^{r}\right)$ we can prove

THEOREM 10. $\exists r$, depending on $k$, so that $\operatorname{Perm}\left(x^{\prime}\right)$ for $x \in D_{k}$ achieves its minimum uniquely at the matrix with equal entries.

Proofs of all the above will appear elsewhere.

\section{REFERENCES}

1. R. A. Brualdi, S. V. Parter and H. Schneider, The diagonal equivalence of a non negative matrix to a stochastic matrix, J. Math. Anal. Appl. 16 (1966), 31-50. MR 34 \# 5844.

2. A. M. Gleason, Remarks on the van der Waerden permanent conjecture, J. Combinatorial Theory 8 (1970), 54-64. MR $41 \# 223$.

3. H. J. Ryser, Permanents and systems of distinct representatives. (With discussion), Combinatorial Mathematics and its Applications (Proc. Conf., Univ. North Carolina, Chapel Hill, N. C., 1967), Univ. North Carolina Press, Chapel Hill, N. C., 1969, pp. 55-70. MR 40 \# 2693.

4. R. Sinkhorn and P. Knopp, Concerning non negative matrices and doubly stochastic matrices, Pacific J. Math. 21 (1967), 343-348. MR 35 \# 1617.

Department of Mathematics, Cornell University, Ithaca, New York 14850 